\title{
A rare triple skeletal bone variation, including a median cleft of the posterior arch of the atlas, a sternal foramina and a bifid xiphoid process
}

\author{
Gregory Tsoucalas, Anastasios Vasilopoulos, Vasilios Thomaidis, Aliki Fiska \\ Department of Anatomy, School of Medicine, Democritus University of Thrace, Alexandroupolis, Greece
}

\begin{abstract}
Bone variations are usually a result of abnormal ossification during embryonic life. Separately or in combination, sternal foramina and bifid xiphoid process are well documented skeletal alternations, as well as the median cleft of the posterior arch of the atlas. However, their appearance in combination is not yet celebrated in the literature. Our post-mortem examination unearthed such a triple variant of an adult male skeleton, which included no other skeletal variations. The fact that all variants are depicted in the median line of ossification implies a hypothesis of a triggered midline bone defect process. The awareness among clinicians of such variations during interventional and imaging procedures is of great importance.
\end{abstract}

Key words: Median cleft of the posterior arch of the atlas, Sternal foramina, Bifid xiphoid process, Abnormal embryonic ossification, Bone variants

Received November 7, 2018; 1st Revised January 14, 2019; 2nd Revised February 6, 2019; Accepted July 5, 2019

\section{Introduction}

The sternum is a flat-shaped bone located in the middle of the anterior surface of the thoracic wall. It comprises of three parts from above downwards, the manubrium, the body or gladiolus, and the xiphoid process. Its embryological development starts from two lateral cartilaginous sternal bars, which fuse in the midline. Sternal ossification process is being completed during postnatal life, between a time interval which begins soon after puberty and ends in the fourth decade of hu-

\section{Corresponding author:}

Gregory Tsoucalas (iD

Department of Anatomy, School of Medicine, Democritus University of Thrace, lerolohiton 155, Agioi Anargyroi 38334, Volos, Greece Tel: +30-2421078583, Fax: +30-2421078160, E-mail: gregorytsoucalas@ yahoo.gr man life. Bar fusion finishes with the formation of the xiphoid process (known also as xiphisternum) which is located in the epigastrium region of the anterior abdominal wall. It articulates with the superiorly located sternum corpus at the xiphisternal joint, which becomes a synostosis at the age of 40 or later [1-3]. Atlas, the first cervical vertebra (C1), supports and holds the globe of the skull at the atlanto-occipital joint. Usually three ossification centers form the atlas. Two ossification centers form the lateral masses, while the median center builds the anterior arch. The ossification proceeds dorsally from the lateral centers to the posterior arch, while the fusion of the ossified atlas parts occurs between 5 to 9 years of age $[4,5]$.

Variations in skeletal anatomy create a difficulty for healthcare professionals in effectively practicing their roles. Sternal foramina (SF) represent a developmental defect, appearing as a result of the incomplete fusion of the sternal bars in the midline. During embryonic life, the incomplete sternal bar fusion explains the occurrence of an SF $[3,6]$. The body of the sternum is the most usual SF location; double foramina are 
occasionally reported [7]. SF presents the risk of spontaneous pneumothorax, vessel injury or pericardial tamponade during sternal biopsy or acupuncture procedures and may be respon-
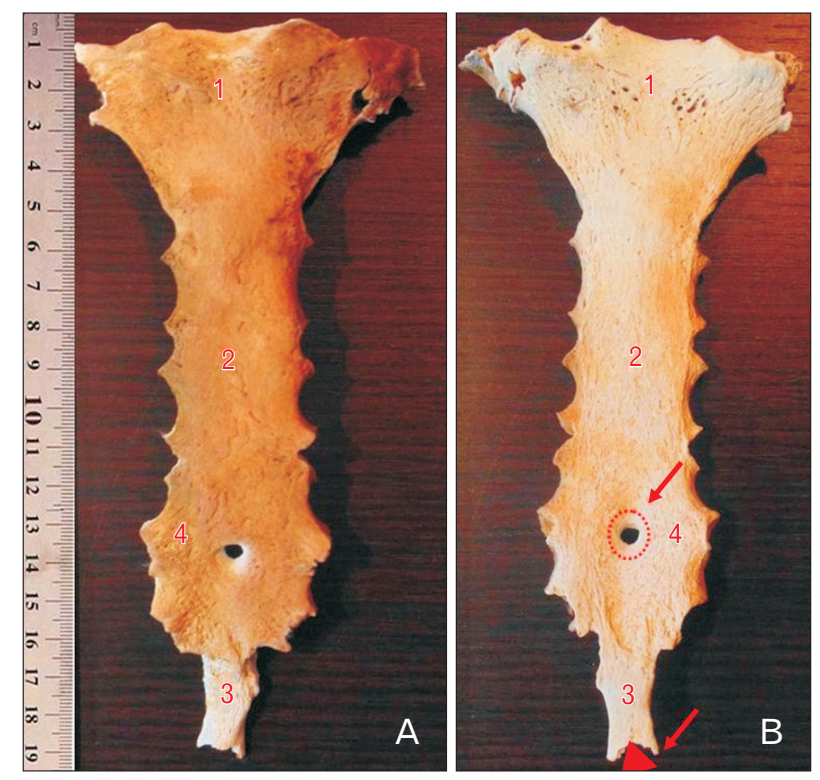

Fig. 1. (A) Sternum bone anterior view. 1, manumbrium sternum; 2, body of the sternum; 3, bifid xiphoid process; 4, sternal foramina. (B) Sernum bone posterior view. 1, manumbrium sternum; 2, body of the sternum; 3, bifid xiphoid process (a triangular indicates the bifid shape); 4, sternal foramina (a circle indicates the round shape). sible for complications during sternotomy. Radiologic misinterpretation of the foramen as an osteolytic lesion could also occur $[3,6]$. The incomplete fusion of the two sternal bars in the caudal body part during embryonic life usually results in a bifid xiphoid process (BXP). A variant of xiphoid morphology, such as the bifid, could be mistaken for a fracture during imaging or even as an epigastric mass. As the BXP is being presented as a midline chondrification failure, is commonly associated with SF [8].

A solitary median cleft of the posterior arch (CPA) of the Atlas may be explained as a failure of chondrogenesis and an ossification anomaly. Its distribution differs between populations, with higher incidence of congenital atlas posterior arch defects in Caucasian population $[4,5]$.

In our case, we demonstrate a triple combination of skeletal defects including SF, BXP, and CPA of the C1, found during a study of a human skeleton donated in our anatomy department.

\section{Case Report}

A human skeleton of Caucasian (Hellenic) origin was examined during routine educational study among the skeletons newly donated at the Anatomy Department of the Medical
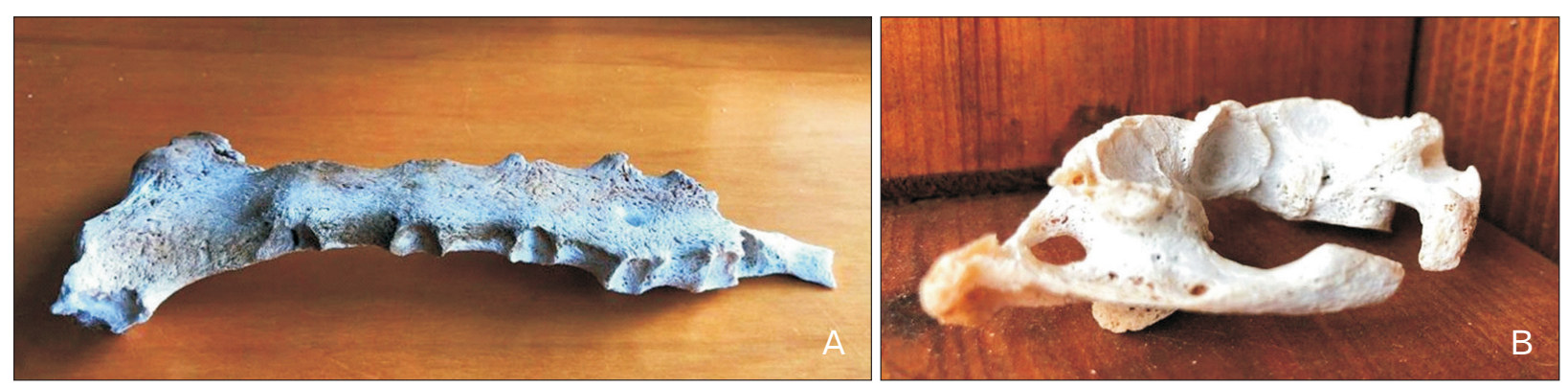

Fig. 2. (A) Stenum bone, lateral view. (B) Atlas bone, dorsal-lateral view.
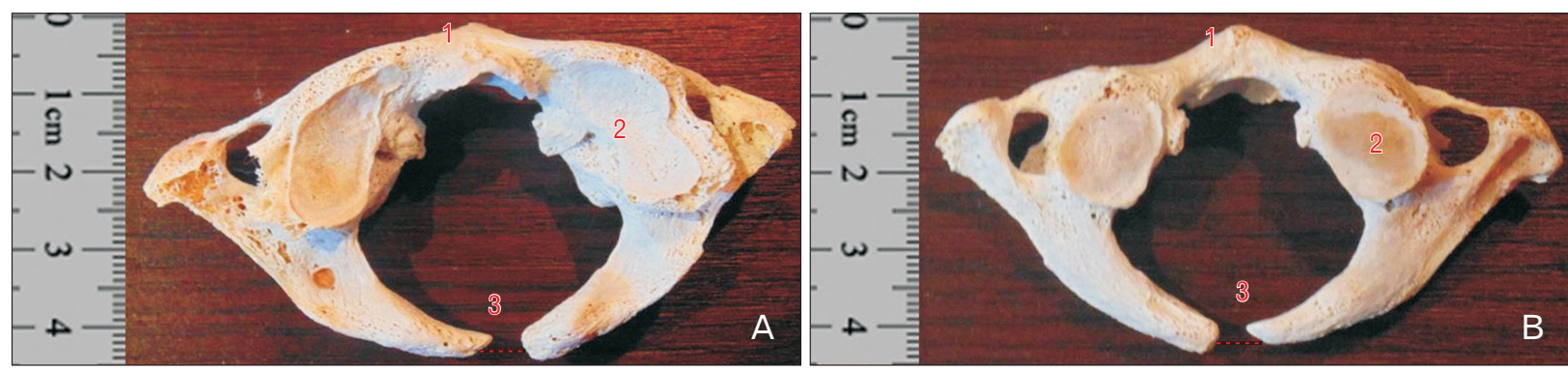

Fig. 3. (A) Atlas bone superior view. 1, anterior tubercle; 2, duperior articular surface; 3, median open cleft of the posterior arch with no posterior tubercle in present. (B) Atlas bone inferior view. 1, anterior tubercle; 2, Inferior articular surface; 3, median open cleft of the posterior arch with no posterior tubercle in present. 
School of the Democritus University of Thrace in Alexandroupolis. The donated skeleton belonged to a 74 years old male. The skeleton derived from skeleton donation with informed consent (with signature authentication) by the donator himself.

Our post-mortem skeletal findings included sternum variations (total sternum length, $19.4 \mathrm{~cm}$; maximum width, $9.4 \mathrm{~cm}$ ) presented with a BXP having a $2.5 \mathrm{~cm}$ length with ends and a SF with a $0.4 \mathrm{~cm}$ vertical and $0.3 \mathrm{~cm}$ horizontal diameter, located at the level of the sixth costal notch (Figs. 1, $2 \mathrm{~A}$ ), combined with a median CPA of the atlas vertebra (3.8 $\mathrm{cm}$ vertical and $7 \mathrm{~cm}$ length) with an opening of $0.3 \mathrm{~cm}$ (total absence of the posterior tubercle (Figs. 2B, 3). The skeleton presented no other bone variations.

\section{Discussion}

A BXP is a congenital variation with a reported incidence up to $43.2 \%$ of the general population [2]. The prevalence of an SF rises to $4.5 \%$, marking this bone defect as a rare one [7]. Congenital spondyloschisis of the $\mathrm{C} 1$ is usually found medially or paramedially in the posterior arch, whereas $97 \%$ of the CPAs tend to be located in the midline with only $3 \%$ occurring laterally $[4,5]$. Bony CPAs of the atlas present an incidence that varies between $0.69 \%$ and $4 \%$ in the literature [4].

Clearly, congenital anomalies in the xiphoid process are not a rare phenomenon, and significant interindividual variation should be expected. SF is also relatively common, as well as the open median CPA of the C1 vertebra [1]. A double occurrence of a BXP and an SF is also noted, considered as a common result of abnormal sternum ossification [9]. However, a triple skeletal variation depicting anomalies in all three bones should be considered as a very rare case; according to our knowledge no such report exists.

All three bone variations of our case are located in the median line of the bones, as well as in the midline of the body. This fact impels us to classify them in the large category of the midline birth defects. However, such a hypothesis needs more research studies and scientific documentation.

Anatomical variations should always be considered during medical diagnostic examinations and invasive procedures, as reaching the correct diagnosis requires thorough study of all defected, fractured or impaired bones. Profound knowledge of skeletal anatomy and its variations seems to be essential for good clinical practice [10].

\section{ORCID}

Gregory Tsoucalas: https://orcid.org/0000-0002-2595-9686

Anastasios Vasilopoulos:

https://orcid.org/0000-0002-3282-8051

Vasilios Thomaidis:

https://orcid.org/0000-0003-1884-7181

Aliki Fiska: https://orcid.org/0000-0003-1787-6116

\section{Author Contributions}

Conceptualization: GT. Data acquisition: GT, AV. Data analysis or interpretation: AF, VT. Drafting of the manuscript: GT. Critical revision of the manuscript: AF, VT. Approval of the final version of the manuscript: all authors.

\section{Conflicts of Interest}

No potential conflict of interest relevant to this article was reported.

\section{References}

1. Standring S. Gray's anatomy: the anatomical basis of clinical practice. 41st ed. New York: Elsevier; 2016.

2. Mashriqi F, D’Antoni AV, Tubbs RS. Xiphoid process variations: a review with an extremely unusual case report. Cureus 2017;9: e1613.

3. Eid S, Iwanaga J, Oskouian RJ, Loukas M, Tubbs RS. Bifid xiphoid process: case report and review. Cureus 2018;10:e3153.

4. Guenkel S, Schlaepfer S, Gordic S, Wanner GA, Simmen HP, Werner CM. Incidence and variants of posterior arch defects of the atlas vertebra. Radiol Res Pract 2013;2013:957280.

5. Ramdhan RC, Palakunnel JJ, Saker E, Araujo MR, Johal J, Loukas M, Tubbs RS. The split atlas anomaly: a comprehensive review. Spine Scholar 2017;1:37-44.

6. El-Busaid H, Kaisha W, Hassanali J, Hassan S, Ogengo J, Mandela P. Sternal foramina and variant xiphoid morphology in a Kenyan population. Folia Morphol (Warsz) 2012;71:19-22.

7. Balta C. An anatomic abnormality: double sternal foramina. Int J Clin Med Images 2018;5:1000605.

8. Singh V. Textbook of clinical embryology. New Delhi: Elsevier Health Sciences; 2017.

9. Rao TR, Rao SR. Presence of unusual foramen in the sternum: a case report. Arch Curr Res Int 2017;10:36511.

10. Romero-Reveron R. Venezuelan surgeons view concerning teaching human anatomical dissection. Anat Cell Biol 2017;50: $12-6$. 